\title{
Review \\ Perspective of Immune Checkpoint Inhibitors in Thymic Carcinoma
}

\author{
Kyoichi Kaira *[D, Hisao Imai (D) and Hiroshi Kagamu \\ Department of Respiratory Medicine, Comprehensive Cancer Center, International Medical Center, \\ Saitama Medical University, 1397-1 Yamane, Hidaka, Saitama 350-1298, Japan; m06701014@gunma-u.ac.jp (H.I.); \\ kagamu19@saitama-med.ac.jp (H.K.) \\ * Correspondence: kkaira1970@yahoo.co.jp; Tel.: +81-42-984-4111; Fax: +81-42-984-4741
}

Citation: Kaira, K.; Imai, H.; Kagamu, H. Perspective of Immune Checkpoint Inhibitors in Thymic Carcinoma. Cancers 2021, 13, 1065. https://doi.org/10.3390/cancers 13051065

Academic Editor: Peter B. Illei

Received: 19 January 2021

Accepted: 25 February 2021

Published: 3 March 2021

Publisher's Note: MDPI stays neutral with regard to jurisdictional claims in published maps and institutional affiliations.

Copyright: (c) 2021 by the authors. Licensee MDPI, Basel, Switzerland. This article is an open access article distributed under the terms and conditions of the Creative Commons Attribution (CC BY) license (https:// creativecommons.org/licenses/by/ $4.0 /)$.
Simple Summary: Thymic carcinoma is a rare neoplasm with a poor outcome, and there are no established therapeutic regimens for metastatic or recurrent disease. Immune checkpoint inhibitors (ICIs), such as PD-1/PD-L1 antibodies, are approved in several human cancers, however, ICIs are not approved in thymic carcinoma. Thus, several clinical trials have been undertaken to demonstrate if they are therapeutically effective for patients with thymic carcinoma. In our review, three prospective phase II studies and several case series were discussed in thymic carcinoma. We found that the objective response rate, disease control rate, and progression-free survival in PD-1 blockade monotherapy were approximately $20 \%, 73 \%$, and four months, respectively. The therapeutic efficacy of PD-1 blockade monotherapy is still limited in patients with thymic carcinoma. Future perspectives focus on the therapeutic implication of tyrokinase inhibitors plus ICIs or new experimental agents plus ICIs alongside several ongoing experimental studies.

Abstract: Thymic carcinoma is a rare neoplasm with a dismal prognosis, and there are no established therapeutic regimens for metastatic or recurrent disease. Immune checkpoint inhibitors (ICIs), such as PD-1/PD-L1 antibodies, are widely approved in several human cancers, contributing to prolonging survival in thoracic tumors. Thymic carcinoma exhibits histologic properties of squamous cell carcinoma (SQC), and resembles the SQC of the lung. ICIs are not approved in thymic carcinoma. Thus, several clinical trials have been undertaken to demonstrate if they are therapeutically effective for patients with thymic carcinoma. In our review, three prospective phase II studies and several case series were discussed in thymic carcinoma. We found that the objective response rate, disease control rate, and progression-free survival in PD-1 blockade monotherapy were approximately 20\%, 73\%, and four months, respectively. Two exploratory investigations indicated that PD-L1 within tumor cells exhibits a possibility of the therapeutic prediction of PD-1 blockade in thymic carcinoma. Several case reports, alongside their treatment content, have also been reviewed. The therapeutic efficacy of PD-1 blockade monotherapy is still limited in patients with thymic carcinoma. Future perspectives focus on the therapeutic implication of tyrokinase inhibitors plus ICIs or new experimental agents plus ICIs alongside several ongoing experimental studies.

Keywords: thymic carcinoma; immunotherapy; immune checkpoint inhibitor; PD-1 blockade; pembrolizumab; nivolumab

\section{Introduction}

Thymic carcinoma (TC) is a rare neoplasm with a poor outcome. If early detected, surgical resection is suitable for its curability, whereas systemic chemotherapy (platinum-based regimens) is usually indicated for patients with metastatic or recurrent disease. However, chemotherapy yielded limited benefits to the outcome and efficacy of TC. Currently, there are no established regimens for the treatment of patients with advanced TC.

In fact, the assessment of chemotherapeutic agents for TC is limited to single-arm phase II trials or retrospective studies with small samples. Recently, Okuma et al. reported 
a systemic review and pooled analysis of anthracycline-, carboplatin-, or cisplatin-based chemotherapy in patients with TC [1]. Their results revealed that the objective response rates (ORRs) of anthracycline-based and non-anthracycline-based chemotherapy for advanced TC were $41.8 \%$ and $40.9 \%$, respectively $(p<0.91)$, whereas those of cisplatin-based and carboplatin-based chemotherapy were $53.6 \%$ and $32.8 \%$, respectively $(p=0.0029)$ in 206 patients collecting 10 studies. They concluded that cisplatin-based chemotherapy was better in patients with TC than carboplatin-based chemotherapy. Anthracycline-based regimens, such as ADOC (doxorubicin, cisplatin, vincristine, and cyclophosphamide), CODE (adriamycin, cisplatin, vincristine, and etoposide) and carboplatin plus amrubicin, and non-anthracycline-based chemotherapy, such as carboplatin plus paclitaxel, cisplatin plus docetaxel, and cisplatin plus irinotecan, are selected based on the judgment of chiefphysicians. In fact, promising drugs, such as molecular targeting agents or immunotherapy aside from anthracycline or platinum-based regimens, are not available, although clinical trials have been conducted in different countries.

Recently, immune checkpoint inhibitors (ICIs), such as anti-programmed death-1 (PD1)/programmed death ligand-1 (PD-L1) antibodies, have been administered to patients with several different kinds of neoplastic types. In particular, PD-1 blockade (nivolumab, pembrolizumab, and atezolizumab) show significant efficacy in patients with advanced non-small-cell lung cancer (NSCLC). Varying efficacies of these antibodies according to PD-L1 expression within tumor cells have been reported. PD-1 blockade monotherapy, or a combination of PD-1 blockade with platinum-based regimens, have been identified as first-line standard treatments [2-5]. By recent investigation, Kaplan-Meier curves of overall survival (OS) exhibited an estimated 5-year rate of $34.2 \%$ among patients with melanoma, 27.7\% among patients with renal cell carcinoma, and $15.6 \%$ among patients with NSCLC [6]. Although the possibility of long-term survivors after PD-1 blockade treatment has been confirmed in several reports [7,8], ICIs have been identified as a curative modality in limited cancer types.

Unfortunately, the results of several trials could not strongly approve the clinical utilization of PD-1 blockade in patients with metastatic or recurrent TC. Therefore, we reviewed the clinical evidence of ICIs and discussed the perspective of this strategy in such patients.

\section{Materials and Methods}

\subsection{Selection Criteria for Literature}

We researched the papers published in English using the MEDLINE and PubMed databases to identify all reviews, clinical studies, and case reports about the relationship between thymic carcinoma and immune checkpoint inhibitors. The search strategy included articles published between March 2018 and November 2020 using the following keywords: "thymic carcinoma", "immunotherapy", "immune checkpoint inhibitor", "PD1", "nivolumab", "pembrolizumab", "atezolizumab", "durvarumab", and "avelumab". We also researched the abstracts to identify reports of low activity that would not appear in the published literature. The search did not restrict the type of publication or periodical. We did not include preliminary results published as abstracts or meeting proceedings. We selected all published reports describing the clinical significance of immune checkpoint inhibitors in TC.

\subsection{Study Selection}

We found 12,044 papers from searching "thymic carcinoma", 372 papers from searching the combination of "thymic carcinoma" and "immunotherapy", 46 papers from searching the combination of "thymic carcinoma" and "immune checkpoint inhibitor", 46 papers from searching the combination of "thymic carcinoma" and "PD-1", 23 papers from searching the combination of "thymic carcinoma" and "pembrolizumab", 11 papers from searching the combination of "thymic carcinoma" and "nivolumab", 0 papers from searching the combination of "thymic carcinoma" and "durvarumab", and 3 papers from searching 
"thymic carcinoma" and "avelumab". Criteria for exclusion were insufficient data on constructed $2 \times 2$ contingency tables and duplicate studies on the same patients. The final decision about inclusion was based on the full article.

\section{Results}

This section may be divided by subheadings. It should provide a concise and precise description of the experimental results, their interpretation, as well as the experimental conclusions that can be drawn.

\subsection{Demographics of Selected Literature}

Among 372 papers, 15 papers were selected as novel reports for our review. There were three review articles, four original articles, six case reports, and two others.

\subsection{Prospective Study}

Three prospective clinical trials focused on 10 patients with thymic carcinoma receiving PD-1 blockade monotherapy [9-11]. The therapeutic demographics of these clinical trials are listed in Table 1.

Table 1. Demographics of prospective phase 2 study of PD-1 blockade in thymic carcinoma.

\begin{tabular}{|c|c|c|c|}
\hline Different Variables & Cho et al. [9] & Giaccone et al. [10] & Katsuya et al. [11] \\
\hline Patient's number & 26 & 40 & 15 \\
\hline $\begin{array}{l}\text { Median age } \\
\text { years (range) }\end{array}$ & 57 years $(26-78)$ & 57 years $(25-80)$ & 55 years $(34-70)$ \\
\hline $\begin{array}{c}\text { Gender } \\
\text { Male/Female }\end{array}$ & $18 / 8(69.2 \% / 30.8 \%)$ & $28 / 12(70 \% / 30 \%)$ & $12 / 3(80 \% / 20 \%)$ \\
\hline $\begin{array}{c}\text { Race } \\
\text { Asian } \\
\text { Caucasian } \\
\text { others }\end{array}$ & $\begin{array}{c}26(100 \%) \\
0(0 \%) \\
0(0 \%)\end{array}$ & $\begin{array}{c}4(10 \%) \\
33(82 \%) \\
3(8 \%)\end{array}$ & $\begin{array}{c}15(100 \%) \\
0(0 \%) \\
0(0 \%)\end{array}$ \\
\hline $\begin{array}{c}\text { ECOG PS } \\
0-1 / 2\end{array}$ & $22(100 \%) / 0(0 \%)$ & $38(96 \%) / 2(5 \%)$ & $15(100 \%) / 0(0 \%)$ \\
\hline Prior use of chemotherapy & $26(100 \%)$ & $40(100 \%)$ & $15(100 \%)$ \\
\hline $\begin{array}{l}\text { ORR } \\
(\%)\end{array}$ & $19.2 \%$ & $22.5 \%$ & $0.0 \%$ \\
\hline $\begin{array}{l}\mathrm{DCR} \\
(\%)\end{array}$ & $73.1 \%$ & $75.0 \%$ & $73.3 \%$ \\
\hline $\begin{array}{c}\text { PFS } \\
\text { (months) }\end{array}$ & 6.1 months & 4.2 months & 3.8 months \\
\hline $\begin{array}{c}\text { OS } \\
\text { (months) }\end{array}$ & 14.5 months & 24.9 months & 14.1 months \\
\hline $\begin{array}{c}\text { 1-year PFS rate } \\
(\%)\end{array}$ & NA & $29.0 \%$ & $9.0 \%$ \\
\hline $\begin{array}{c}\text { 1-year OS rate } \\
(\%)\end{array}$ & NA & $71.0 \%$ & $60.0 \%$ \\
\hline Previous radiotherapy & $12(46.2 \%)$ & $23(58 \%)$ & $7(73.3 \%)$ \\
\hline Curative surgery & $9(34.6 \%)$ & $21(52 \%)$ & $3(20 \%)$ \\
\hline $\begin{array}{l}\text { irAEs } \\
\text { Grade 1-2 } \\
\text { Grade 3-4 }\end{array}$ & $\begin{array}{c}\text { Thyroiditis }(3.8 \%) \\
\text { Pruritus }(11.5 \%) \\
\text { Skin rash }(7.7 \%) \\
\text { Hepatitis }(7.7 \%) \\
\text { Myasthenia gravis }(7.7 \%) \\
\text { Subacute myoclonus }(3.8 \%)\end{array}$ & $\begin{array}{c}\text { Fever }(13.8 \%) \\
\text { Hypothyroidism }(13 \%) \\
\text { Rash }(10 \%) \\
\text { ALT / AST increased (13\%) } \\
\text { Myocarditis (5\%) } \\
\text { Myalgia or Myositis (8\%) }\end{array}$ & $\begin{array}{c}\text { Rash (27\%) } \\
\text { Hypothyroidism (7\%) } \\
\text { Diarrhea (20\%) } \\
\text { AST increased (7\%) }\end{array}$ \\
\hline
\end{tabular}

Abbreviation: PD-1, programmed death-1; ECOG, eastern clinical oncology group; PS, performance status; ORR, objective response rate; PFS, progression-free survival; OS, overall survival; irAEs, immune-related adverse events; ALT, alanine aminotransferase; AST, aspartate aminotransferase; NA, not applicable. 
Pembrolizumab: Cho et al. conducted a phase II study to evaluate the overall response rate (ORR) of pembrolizumab as the primary endpoint in 33 patients with refractory or relapsed thymic epithelial tumors [9]. Among the 33 patients, 26 had TC, and 7 had thymoma. The ORR and disease control rate (DCR) of the thymoma cases were $28.6 \%$ and $100 \%$, respectively, while the ORR, DCR, median progression-free survival (PFS), and median overall survival (OS) in TC were 19.2\%, 73.1\%, 6.1 months, and 14.5 months, respectively. In TC patients, $12(46.2 \%)$ had already received radiotherapy for the primary mediastinal mass, $9(34.6 \%)$ underwent curative-intent surgery for the tumor, and $11(42.3 \%)$ were treated with pembrolizumab as a first-line treatment, after previous chemotherapy. All enrolled patients were Korean. Immune-related adverse events (irAEs) in the TC patients included grade 1-2 thyroiditis, pruritus, skin rash, grade 3-4 hepatitis, myasthenia gravis, and subacute myoclonus in $1(3.8 \%), 3(11.5 \%), 2(7.7 \%), 2$ (7.7\%), 2 (7.7\%), and $1(3.8 \%)$, respectively. In addition, they examined the relationship between the efficacy of pembrolizumab and PD-1 immunostaining in 24 patients (72.7\%); however, little is known about the number of patients with TC histology. High PD-L1 expression $(\geq 50 \%$ tumor proportion score) was observed in $14(58.3 \%)$ patients. Among the 24 patients with high PDL1 expression, 5 (35.7\%) achieved partial response (PR), whereas none of the patients with low PD-L1 expression yielded a response $(p=0.034)$. There was no association between the frequency of irAEs and the status of PD-L1 expression. This study found a significant correlation between PD-L1 protein expression and mRNA expression. The results of this study indicated that pembrolizumab monotherapy is active and tolerable with manageable irAEs for patients with advanced TC, and the expression level of PD-L1 may predict the efficacy of pembrolizumab using an exploratory approach.

Giaccone et al. also reported a phase 2 study to evaluate the efficacy of pembrolizumab in patients with TC [10]. In their study, 40 patients were eligible, and the median follow-up period was 20 months. Nineteen ( $48 \%$ ) had a histology of squamous cell carcinoma, four $(10 \%)$ were Asian, and $33(82 \%)$ were Caucasian. Twenty-three (58\%) patients received previous chest radiotherapy, and $21(52 \%)$ were treated with a previous thymectomy. The ORR and DCR yielded $22.5 \%$ and $75.0 \%$, respectively. One patient achieved a complete response (CR), 8 (20\%) PR, 21 (53\%) stable disease (SD), and 10 progressive disease (PD), without any cases of pseudoprogression. The median PFS, median OS, 1-year PFS, and 1-year OS were 4.2 months, 24.9 months, $29 \%$ and $71 \%$, respectively. The most common grade 3 or 4 adverse events were increased aspartate aminotransferase and alanine aminotransferase (13\% patients, each). Moreover, six (15\%) patients developed one or more severe irAEs. In particular, $2(5 \%)$ patients experienced polymyositis and myocarditis, requiring high-dose steroid therapy. In this study, PD-L1 immunostaining was investigated in 37 patients. High PD-L1 expression ( $\geq 50 \%$ tumor proportion score) was observed in $10(25 \%)$ patients, 6 of whom achieved a PR or CR (5 PR and 1 CR) $(p=0.005)$. Among the 27 patients with low PD-L1 expression, 23 had PD. Their post-hoc analysis demonstrated that median PFS was longer in patients with high PD-L1 expression than in those with low expression (24 months vs. 2.9 months), and OS in patients with high expression was longer than in those with low expression (not reached vs. 15.5 months). In subsequent analysis, 5 of 40 eligible patients completed two years of treatment, and four of them elected to continue treatment, with more than four years [11].

Nivolumab: Katsuya et al. reported a phase II study of nivolumab in 15 patients with unresectable or recurrent TC to evaluate ORR as the primary endpoint [12]. Thirteen patients had a histology of squamous cell carcinoma. Seven $(46.6 \%)$ patients received prior radiotherapy, and prior surgery was performed in three $(20 \%)$ patients. The median follow-up period was 14.1 months. The ORR, DCR, median PFS, and median OS were $0 \%, 73.3 \%, 3.8$ months, and 14.1 months, respectively. Eleven patients achieved SD, and four had PD. The subgroup analysis of 13 patients with squamous cell carcinoma indicated that the median PFS and OS were 3.8 months and 14.1 months, respectively. Moreover, the median PFS and OS in seven patients with previous radiation therapy were 3.8 months and 12.7 months, respectively. The profile of adverse events was mild consistent with previous 
data. Among the two patients who experienced serious irAEs, grade 3 transaminase increase was observed in one, and grade 2 adrenal insufficiency was observed in the other requiring admission.

\subsection{Exploratory Study}

Ak et al. explored their experience with nivolumab for metastatic thymic epithelial tumors [13]. Among the 46 unresectable and recurrent thymic epithelial tumors (32 thymoma and 14 TC), 8 ( 3 TC, 4 thymoma, and one mixed histology of thymoma and TC) received nivolumab. Table 2 shows the clinical features of four patients with TC. Among them, two achieved an ORR of PR, but the median PFS of these two patients was 2.0 and 5.8 months, respectively. There was PD-L1 expression with more than $50 \%$. Among them, one patient experienced severe irAEs, such as dyslexia and motor neuropathy, requiring the withdrawal of nivolumab.

Table 2. Clinical features of four patients with thymic carcinoma (exploratory study).

\begin{tabular}{|c|c|c|c|c|c|c|c|c|c|c|c|c|}
\hline \multirow[b]{2}{*}{ Patient } & \multirow[b]{2}{*}{ Gender } & \multirow{2}{*}{ Age } & \multirow{2}{*}{ Histology } & \multirow{2}{*}{$\begin{array}{c}\text { PD-L1 } \\
(\%)\end{array}$} & \multirow{2}{*}{$\begin{array}{l}\text { Treatments } \\
\text { before ICI }\end{array}$} & \multicolumn{4}{|c|}{ Nivolumab Treatment } & \multirow{2}{*}{$\begin{array}{c}\text { Survival } \\
\text { before ICI } \\
\text { (Months) }\end{array}$} & \multirow{2}{*}{$\begin{array}{l}\text { Survival } \\
\text { after ICI } \\
\text { (Months) }\end{array}$} & \multirow{2}{*}{$\begin{array}{l}\text { Total OS } \\
\text { (Months) }\end{array}$} \\
\hline & & & & & & $\begin{array}{l}\text { Total N of } \\
\text { Cycles }\end{array}$ & $\begin{array}{c}\text { Best } \\
\text { Response }\end{array}$ & $\begin{array}{c}\text { PFS } \\
\text { (Months) }\end{array}$ & irAEs & & & \\
\hline No. 1 & M & 60 & SCC & 60 & $\begin{array}{c}\text { Pac/Carbo, } \\
\text { Etoposide, } \\
\text { CAP }\end{array}$ & 3 & SD & 3.2 & none & 41.2 & 22.1 & 63.3 \\
\hline No. 2 & M & 26 & $\begin{array}{c}\mathrm{SCC}, \\
\mathrm{T}\end{array}$ & 100 & Pac/Carbo & 3 & PR & 2.0 & $\begin{array}{l}\text { Myasthenic } \\
\text { Symptoms } \\
\text { Grade } 1\end{array}$ & 70.2 & 6.5 & 76.7 \\
\hline No. 3 & F & 67 & SCC & 100 & CAP & 1 & NA & NA & $\begin{array}{c}\text { Dislexia, } \\
\text { motor } \\
\text { neuropathy } \\
\text { Grade } 4\end{array}$ & 7.5 & 6.6 & 14.1 \\
\hline No. 4 & $F$ & 73 & SCC & 50 & Pac/Carbo & 10 & PR & 5.8 & none & 10.5 & 6.2 & 16.7 \\
\hline
\end{tabular}

Abbreviations: PD-L1, programmed death ligand-1; ICI, immune checkpoint inhibitor; N, number; PFS, progression-free survival; OS, overall survival; SCC, squamous cell carcinoma; T, thymoma; Pac, paclitaxel; Carbo, carboplatin; CAP, cisplatin/adriamisin/cyclophosphamide; $\mathrm{SD}$, stable disease; PR, partial response; NA, not applicable. Nivolumab was administered intravenously at $3 \mathrm{mg} / \mathrm{kg}$ or $240 \mathrm{mg} / \mathrm{day}$ every two weeks.

\subsection{Case Reports}

We found five case reports indicating the unique clinical course of patients with TC who received PD-1 blockade [14-18]. The summary of these case reports is displayed in Table 3.

Jin et al. reported two cases of refractory thymic squamous cell carcinoma receiving nivolumab or pembrolizumab combined with chemotherapy/angiogenetic therapy [14]. Case 1 received nivolumab as a second-line therapy, sequentially, re-administration of nivolumab combined with nab-paclitaxel as a fifth-line therapy. The OS from initiation of nivolumab was more than three years. Case 2 was treated with pembrolizumab as a second-line therapy, with PD. Sequentially, combination therapy with anlotinib plus pembrolizumab was continued for 16 cycles with SD. After PD, the patient experienced PR, switching to combination with pembrolizumab plus gemcitabine. The duration of pembrolizumab and its combination therapy was 23 months. Cafaro et al. described a case of heavily pretreated TC who was treated with pembrolizumab as the fourth-line treatment [15]. Uchida et al. demonstrated an experience of nivolumab in four cases of unresectable TC [16]. Case 1 was treated with carboplatin plus nab-paclitaxel as a first-line treatment, and radiotherapy was initiated as palliative therapy. The patient then received nivolumab as a second-line therapy, and PR was found after five cycles. Case 2 also received nivolumab after first-line treatment with carboplatin plus nab-paclitaxel. After four cycles of nivolumab, there was evidence of PR. In case 3, carboplatin plus paclitaxel, as a first-line treatment, was continued until four cycles, then sequentially with gemcitabine for 44 cycles. Nivolumab was administered as a third-line treatment, and radiological assessment showed PR after four cycles. Case 4 received five cycles of carboplatin plus paclitaxel as a first-line therapy and six cycles of gemcitabine as a second-line therapy. 
Nivolumab was chosen as a third-line treatment, and six cycles were completed with a response of SD. Yang et al. described a better response to nivolumab as a fourth-line therapy in a patient with TC [17]. Isshiki et al. documented a successful case of refractory thymic carcinoma with high PD-L1 expression treated with pembrolizumab as a second-line therapy after three cycles of carboplatin plus nab-paclitaxel [18].

Table 3. Summary of case series regarding PD-1 blockade treatment in thymic carcinoma.

\begin{tabular}{|c|c|c|c|c|c|c|}
\hline Case Series & Age & Sex & Treatment Line & $\begin{array}{l}\text { PD-1 Blockade or Combination } \\
\text { Regimens (Response) }\end{array}$ & $\begin{array}{l}\text { Complete Cycles or } \\
\text { Treatment Duration }\end{array}$ & irAEs \\
\hline \multicolumn{7}{|c|}{ Jin et al. [14] } \\
\hline Case 1 & 40 & $\mathrm{M}$ & $\begin{array}{l}\text { 2nd } \\
5 \text { th }\end{array}$ & $\begin{array}{c}\text { Nivolumab (SD) } \\
\text { Nivolumab plus nab-paclitaxel (PR) }\end{array}$ & $\begin{array}{l}40 \text { cycles }^{\dagger} \\
38 \text { months } \\
3 \text { cycles }\end{array}$ & G1 skin \\
\hline \multirow{3}{*}{ Case 2} & \multirow{3}{*}{52} & \multirow{3}{*}{ M } & 2nd & Pembrolizumab (PD) & 2 cycles $\S$ & \multirow{3}{*}{ Gr3 skin } \\
\hline & & & $3 \mathrm{rd}$ & Pembrolizumab plus anlotinib (SD) & 16 cycles & \\
\hline & & & 4 th & Pembrolizumab plus gemcitabine (PR) & 23 months $\ddagger$ & \\
\hline \multirow{3}{*}{ Case 1} & \multicolumn{5}{|c|}{ Cafaro et al. [15] } & \multirow[b]{2}{*}{ NA } \\
\hline & 57 & M & 4th & Pembrolizumab (SD) & 18 cycles & \\
\hline & \multicolumn{5}{|c|}{ Uchida et al. [16] } & \multirow[b]{2}{*}{ NA } \\
\hline Case 1 & 72 & $\mathrm{~F}$ & 2nd & Nivolumab (PR) & NA & \\
\hline Case 2 & 64 & $\mathrm{~F}$ & 2nd & Nivolumab (PR) & NA & NA \\
\hline Case 3 & 66 & $\mathrm{M}$ & $3 \mathrm{rd}$ & Nivolumab (PR) & 8 cycles & NA \\
\hline Case 4 & 72 & $\mathrm{~F}$ & $3 r d$ & Nivolumab (PR) & 6 cycles & NA \\
\hline \multicolumn{7}{|c|}{ Yang et al. [17] } \\
\hline Case 1 & 62 & $\mathrm{~F}$ & 4 th & Nivolumab (PR) & NA & G1 skin \\
\hline \multicolumn{7}{|c|}{ Isshiki et al. [18] } \\
\hline
\end{tabular}

Abbreviation: PD-1, programmed death-1; irAEs, immune-related adverse events; G, grade; SD, stable disease; PR, partial response; PD, progressive disease; NA, not available; M, male; F, female; 1st, 1st line treatment; 2nd, 2nd line treatment, 3rd, 3rd line treatment; 4th, 4th line treatment. ${ }^{\dagger}$ Nivolumab and $\S$ pembrolizumab were administered intravenously at $3 \mathrm{mg} / \mathrm{kg}$ or $240 \mathrm{mg} /$ day every two weeks and $200 \mathrm{mg} /$ day every three weeks, respectively. ${ }^{\ddagger}$ This time is defined as time to progressive disease.

\subsection{Clinicopathological Relevance of PD-L1 Expression in Thymic Carcinoma}

The expression of PD-L1 within tumor cells is associated with the therapeutic prediction of PD-1 blockade in human cancers, especially NSCLC or head and neck cancer $[2,19,20]$. Although several descriptions regarding the relationship between prognostic significance and PD-L1 expression in patients with different types of cancers have already been reported, several researchers also documented whether the expression of PD-L1 could be related to tumor aggressiveness and survival in patients with TC [21-26]. Recently, Katsuya et al. reported the prognostic relevance of PD-L1 expression in patients with 102 thymomas and 37 thymic carcinomas [21]. The positive rate of PD-L1 expression was identified as $70 \%$ in TC and $23 \%$ in thymoma, with a significant difference $(p<0.001)$. However, there was no significant difference in OS between positive and negative PD-L1 expression in TC. In another report, high expression of PD-L1 was observed in 25 (36\%) patients with TC, though no significant difference in the PFS and OS was observed between patients with high and low PD-L1 expression [22]. Weissferdt et al. also did not find any prognostic impact of high PD-L1 expression, although expression of PD-L1 was recognized in $14(54 \%)$ patients with TC [23]. Moreover, a clinicopathological study of 20 patients with TC revealed a trend of better OS in patients with low PD-1 expression, without a significant difference [24]. On the other hand, Yokoyama et al. demonstrated the opposite results of the prognostic relevance of PD-L1 expression compared to those of several studies above [25]. In their study, PD-L1 was highly expressed in $20(80 \%)$ patients with TC, and low PD-L1 expression was identified as a significant predictor of worse survival [24]. However, Funaki et al. reported that PD-L1 was highly expressed in 17 (39.5\%) patients with TC, and positive PD-L1 expression was closely associated with worse PFS and OS after surgical 
resection [26]. To date, some studies have analyzed the prognostic relevance of PD-L1 expression in all patients with TC and thymoma $[27,28]$. The expression level of PD-L1 displayed a higher trend in TC than in thymoma; however, they appeared to be conflicting results regarding whether PD-L1 could predict better or worse outcomes. Thymoma complicates autoimmune disorders (myasthenia gravis) and includes immature T-cells expressing terminal deoxynucleotidyl transferase in tumor tissues, whereas TC is known to lack immature T-lymphocytes and does not induce autoimmune diseases [29,30]. As the tumor immune environment is different between thymoma and TC and TC also exhibited significantly worse survival than thymoma, both diseases should be distinguished in the survival analysis. From previous studies, we found that PD-L1 is highly expressed in TC, but it remains unclear whether the expression level of PD-L1 predicts the outcome after any treatment. The difference in the clone of PD-L1 antibody for immunohistochemistry and study design may have biased the results of each investigation. Although a small sample size is dominant owing to the rarity of the disease, further study is required to evaluate the prognostic implications of PD-L1 expression in TC using a large-scale approach. The reagents and scoring criteria used for the assessment of PD-L1 above studies are listed in Table 4.

Table 4. Reagents and scoring criteria used for the assessment of PD-L1.

\begin{tabular}{|c|c|c|c|}
\hline Reference & Clone of PD-L1 AntiBody & Company, City, Country & Definition of Scoring Criteria \\
\hline [9] & $22 \mathrm{C} 3$ & $\begin{array}{l}\text { Dako North America, } \\
\text { Carpinteria, CA, USA }\end{array}$ & $\begin{array}{l}\text { PD-L1 expression was classified as "high" if } \\
\text { at least } 50 \% \text { of the tumor cells, inflammatory } \\
\text { cells, or stroma cells stained positive. PD-L1 } \\
\text { expression in } 0 \% \text { to } 49 \% \text { of cells was } \\
\text { classified as "low" expression. }\end{array}$ \\
\hline [13] & $28-8$ & Abcam, Tokyo, Japan & PD-L1 $\geq 1 \%$ was defined as "positive". \\
\hline [10] & $22 \mathrm{C} 3$ & $\begin{array}{c}\text { Agilent Technologies, Santa } \\
\text { Clara, CA, USA }\end{array}$ & $\begin{array}{l}\text { PD-L1 expression was classified as "high" if } \\
\text { at least } 50 \% \text { of the tumor cells stained } \\
\text { positive. PD-L1 expression in 1-49\% of cells } \\
\text { was classified as "low" expression. }\end{array}$ \\
\hline
\end{tabular}

Abbreviation: PD-L1, programmed death ligand-1.

\section{Discussion}

Currently, PD-1 blockade is approved in patients with several types of human neoplasms. In particular, it has been found to improve the therapeutic efficacy of PD-1 blockade combined with cytotoxic agents or angiogenetic drugs [4]. Therefore, we expect that PD-1 blockade is therapeutically effective for patients with TC as rare cancer. In our review, three prospective phase II studies and several case series regarding TC were discussed, and we found that the ORR, DCR, and median PFS in PD-1 blockade monotherapy were approximately $20 \%, 73 \%$, and four months, respectively. Compared with the clinical trial of nivolumab in previously treated NSCLC, the therapeutic outcome of PD-1 blockade monotherapy in TC seemed favorable, and the increased frequency of irAEs was not found. Although the expression of PD-L1 within tumor cells is known to predict the efficacy of PD-1 blockade in patients with NSCLC, two exploratory investigations indicated that PD-L1 has the potential to precisely predict PD-1 blockade in TC $[9,10]$. Cho et al. and Giaccone et al. described that the clinical benefit according to the expression level of PD-L1 seems to be apparent for predicting the efficacy of PD-1 blockade in patients with thymic carcinoma $[9,10]$. Several case reports have also been reviewed, and the treatment content discussed. In clinical practice, nivolumab or pembrolizumab is active for patients with previously treated TC. Moreover, their clinical effectiveness was confirmed to persist in the long-term. Further studies are required to confirm the efficacy of PD-1 blockade according to the expression level of PD-L1.

To improve the therapeutic efficacy of TC, the development of new regimens, such as tyrosine kinase inhibitors (TKIs), ICIs, and their combination, is necessary, and many 
studies are ongoing. As clinical trials of PD-1 blockade monotherapy in patients with thymic epithelial tumors, three phase II studies are currently ongoing. Atezolizumab was investigated in 34 patients with pretreated TC, with the primary endpoint of ORR and secondary endpoint of PFS, OS, duration of objective response (DOR), DCR, adverse events, and distribution of PD-L1 and TMB expression (NCT04321330). Nivolumab was examined in 55 patients with previously treated TC and thymoma type B3 after platinumbased chemotherapy, with the primary endpoint of PFS rate at six months and secondary endpoint of PFS, OS, ORR, DCR, and DOR (NCT03134118). Avelumab was investigated for 55 patients with previously treated TC and thymoma after platinum-based chemotherapy, with a primary endpoint of ORR and safety and secondary endpoint of immune-related PFS, OS, and DOR (NCT03076554). The PFS, OS, and DOR were common in these three studies, but primary outcome measures were different among these studies, PFS or ORR.

In addition, combination therapy of TKIs with PD-1 blockade has also been examined as a targeted treatment for thymic epithelial tumors. A phase II study evaluating the ORR of pembrolizumab plus sunitinib is currently ongoing in 40 patients with TC who are resistant to platinum-based regimens (NCT03463460). The primary objective of this study is ORR and secondary objectives are to evaluate safety profile, PFS and OS. As exploratory investigations, it is planning to determine whether sunitinib leads to an increase in PDL1 expression, TILs, and decrease in myeloid-derived suppressor cells (MDSC) in both tumor and peripheral blood. In the CAVEATT study, 33 patients with TC and thymoma type B3 treated with platinum-based chemotherapy are registered and received avelumab combined with axitinib (an oral VEGFR-1/2/3 kinase inhibitor) for the primary endpoint of ORR. In phase I/II trials, nivolumab plus vorolanib, an oral VEGFR/PDGFR kinase inhibitor, is in progress for the aim of safety and ORR in 177 patients with pretreated thoracic tumors, including TC (NCT03583086). The primary objectives of this study are safety and tolerability of nivolumab plus vorolanib as a phase I study and efficacy as a phase II study, and secondary objectives are PFS and OS. Finally, pembrolizumab with or without SC-C101 (a superagonist fusion protein of interleukin-15) is currently ongoing as a phase $1 / \mathrm{Ib}$ study for 96 patients with pretreated solid tumors, including thymic epithelial tumors (NCT04234113). The primary objective of this study is to determine dose-limiting toxicities and treatment-related adverse events, and secondary objectives are ORR, BOR, PFS, and clinical benefit rate. These clinical trials are promising and are expected to provide future perspectives.

\section{Conclusions}

Very few prospective studies have evaluated the efficacy of PD-1 blockade monotherapy (pembrolizumab or nivolumab) in patients with pretreated TC. The therapeutic efficacy of PD-1 blockade monotherapy is still limited in such patients, similar to the therapeutic results of advanced NSCLC. Although it remains unclear whether PD-L1 expression could predict the efficacy of PD-1 blockade monotherapy in TC, an exploratory investigation suggests an increased response of PD-1 blockade in patients with high PD-L expression. We believe that PD-L1 appears predictive in such patients. Future perspectives focusing on the therapeutic implication of TKIs plus ICIs or new experimental agents plus ICIs, and several experimental studies are currently ongoing.

Funding: This research received no external funding.

Institutional Review Board Statement: Not applicable.

Informed Consent Statement: Not applicable.

Data Availability Statement: No new data were created or analyzed in this study. Data sharing is not applicable to this article.

Conflicts of Interest: K.K. has received research grants and is a speaker honorarium from Ono Pharmaceutical Company, Boehringer Ingelheim, Chugai Pharmaceutical, Taiho Pharmaceutical, Eli Lilly Japan, and AstraZeneca. H.K. has received research grants and a speaker honorarium from 
Ono Pharmaceutical Company, Bristol-Myers Company, Boehringer Ingelheim, MSD, Daiichi Sankyo Company, Chugai Pharmaceutical, Taiho Pharmaceutical, Merck Biopharma Company, Eli Lilly Japan, and AstraZeneca.

\section{References}

1. Okuma, Y.; Saito, M.; Hosomi, Y.; Sakuyama, T.; Okamura, T. Key components of chemotherapy for thymic malignancies: A systematic review and pooled analysis for anthracycline-, carboplatin- or cisplatin-based chemotherapy. J. Cancer Res. Clin. Oncol. 2015, 141, 323-331. [CrossRef]

2. Reck, M.; Rodríguez-Abreu, D.; Robinson, A.G.; Hui, R.; Csőszi, T.; Fülöp, A.; Gottfried, M.; Peled, N.; Tafreshi, A.; Cuffe, S.; et al. Pembrolizumab versus chemotherapy for PD-L1-positive non-small-cell lung cancer. N. Engl. J. Med. 2016, 375, 1823-1833. [CrossRef] [PubMed]

3. Gandhi, L.; Rodríguez-Abreu, D.; Gadgeel, S.; Esteban, E.; Felip, E.; De Angelis, F.; Domine, M.; Clingan, P.; Hochmair, M.J.; Powell, S.F.; et al. Pembrolizumab plus chemotherapy in metastatic non-small-cell lung cancer. N. Engl. J. Med. 2018, 378, 2078-2092. [CrossRef]

4. Socinski, M.A.; Jotte, R.M.; Cappuzzo, F.; Orlandi, F.; Stroyakovskiy, D.; Nogami, N.; Rodríguez-Abreu, D.; Moro-Sibilot, D.; Thomas, C.A.; Barlesi, F.; et al. Atezolizumab for first-line treatment of metastatic non-squamous NSCLC. N. Engl. J. Med. 2018, 378, 2288-2301. [CrossRef] [PubMed]

5. Carbone, D.P.; Reck, M.; Paz-Ares, L.; Creelan, B.; Horn, L.; Steins, M.; Felip, E.; van den Heuvel, M.M.; Ciuleanu, T.E.; Badin, F.; et al. First-line Nivolumab in Stage IV or recurrent non-small-cell lung cancer. N. Engl. J. Med. 2017, 376, 2415-2426. [CrossRef]

6. Topalian, S.L.; Hodi, F.S.; Brahmer, J.R.; Gettinger, S.N.; Smith, D.C.; McDermott, D.F.; Powderly, J.D.; Sosman, J.A.; Atkins, M.B.; Leming, P.D.; et al. Five-year Survival and Correlates among Patients with Advanced Melanoma, Renal Cell Carcinoma, or Non-Small Cell Lung Cancer Treated with Nivolumab. JAMA Oncol. 2019, 5, 1411-1420. [CrossRef]

7. Gettinger, S.; Horn, L.; Jackman, D.; Spigel, D.; Antonia, S.; Hellmann, M.; Powderly, J.; Heist, R.; Sequist, L.V.; Smith, D.C.; et al. Five-year Follow-Up of Nivolumab in Previously Treated Advanced Non-Small-Cell Lung Cancer: Results from the CA209-003 Study. J. Clin. Oncol. 2018, 6, 1675-1684. [CrossRef] [PubMed]

8. Garon, E.B.; Hellmann, M.D.; Rizvi, N.A.; Carcereny, E.; Leighl, N.B.; Ahn, M.J.; Eder, J.P.; Balmanoukian, A.S.; Aggarwal, C.; Horn, L.; et al. Five-year overall survival for patients with advanced non-small-cell lung cancer treated with Pembrolizumab: Results from the Phase I KEYNOTE-001 study. J. Clin. Oncol. 2019, 37, 2518-2527. [CrossRef] [PubMed]

9. Cho, J.; Kim, H.S.; Ku, B.M.; Choi, Y.L.; Cristescu, R.; Han, J.; Sun, J.M.; Lee, S.H.; Ahn, J.S.; Park, K.; et al. Pembrolizumab for patients with refractory on relapsed thymic epithelial tumor: An open-label phase II trial. J. Clin. Oncol. 2019, 37, $2162-2170$. [CrossRef] [PubMed]

10. Giaccone, G.; Kim, C.; Thompson, J.; McGuire, C.; Kallakury, B.; Chahine, J.J.; Manning, M.; Mogg, R.; Blumenschein, W.M.; Tan, M.T.; et al. Pembrolizumab in patients with thymic carcinoma: A single-arm, single-centre, phase 2 study. Lancet Oncol. 2018, 19, 347-355. [CrossRef]

11. Giaccone, G.; Kim, C. Durable response in patients with thymic carcinoma treated with pembrolizumab after prolonged follow-up. J. Thorac. Oncol. 2020. [CrossRef]

12. Katsuya, Y.; Horinouchi, H.; Seto, T.; Umemura, S.; Hosomi, Y.; Satouchi, M.; Nishio, M.; Kozuki, T.; Hida, T.; Sukigara, T.; et al. Single-arm, multicenter, phase II trial of nivolumab for unresectable or recurrent thymic carcinoma: PRIMER study. Eur. J. Cancer 2019, 113, 78-86. [CrossRef]

13. Ak, N.; Aydiner, A. Nivolumab treatment for metastatic thymic epithelial tumors. J. Oncol. Pharm. Pract. 2020. [CrossRef]

14. Jin, W.; Duan, J.C.; Wang, Z.J.; Lin, L.; Bai, H.; Wang, J.; Feng, L. The effect and safety of anti-PD-1 single/combination therapy in refractory thymic carcinoma: A case-series study. Cancer Manag. Res. 2020, 12, 11351-11358. [CrossRef] [PubMed]

15. Cafaro, A.; Bongiowanni, A.; Iorio, V.D.; Oboldo, D.; Masini, C.; Ibarahim, T. Pembrolizumab in a patients with heavily pre-treated squamous cell thymic carcinoma and cardiac impairment: A case report and literature review. Front. Oncol. 2020, 10, 1478. [CrossRef] [PubMed]

16. Uchida, N.; Fujita, K.; Okamura, M.; Nakatani, K.; Mio, T. The clinical benefits of immune checkpoint inhibitor for thymic carciniomas experience of single public hospital in Japan. Respir. Med. Case Rep. 2018, 26, 39-41.

17. Tang, P.C.; Guo, J.C.; Hsieh, M.S.; Lin, C.C.; Hsu, C.H. Response to nivolumab as salvage therapy in a patients with thymic carcinoma. J. Thorac. Oncol. 2018, 13, e36-e39.

18. Isshiki, T.; Isobe, K.; Tochigi, N.; Sunakawa, M.; Nakamura, Y.; Shibuya, K.; Sakamoto, S.; Takai, Y.; Homma, S. Successful use of pembrolizumab to treat refractory thymic carcinoma with high PD-L1 expression. Case Rep. Oncol. 2018, 11, 688-692. [CrossRef]

19. Garon, E.B.; Rizvi, N.A.; Hui, R.; Leighl, N.; Balmanoukian, A.S.; Eder, J.P.; Patnaik, A.; Aggarwal, C.; Gubens, M.; Horn, L.; et al. Pembrolizumab for the treatment of non-small-cell lung cancer. N. Engl. J. Med. 2015, 372, 2018-2028. [CrossRef]

20. Ferris, R.L.; Blumenschein, G., Jr.; Fayette, J.; Guigay, J.; Colevas, A.D.; Licitra, L.; Harrington, K.; Kasper, S.; Vokes, E.E.; Even, C.; et al. Nivolumab for recurrent squamous-cell carcinoma of the head and neck. N. Engl. J. Med. 2016, 375, 1856-1867. [CrossRef]

21. Katsuya, Y.; Fujita, Y.; Horinouchi, H.; Ohe, Y.; Watanabe, S.; Tsuta, K. Immunohistochemical status of PD-L1 in thymoma and thymic carcinoma. Lung Cancer 2015, 88, 154-159. [CrossRef]

22. Wei, Y.F.; Chu, Y.C.; Chang, C.C.; Lin, S.H.; Su, W.C.; Tseng, Y.L.; Lin, C.C.; Yen, Y.T. Different pattern of PD-L1, IDO, and FOXP3 Tregs expression with survival in thymoma and thymic carcinoma. Lung Cancer 2018, 125, 35-42. [CrossRef] [PubMed] 
23. Weissferdt, A.; Fujimoto, J.; Kalhor, N.; Rodriguez, J.; Bassett, R.; Wistuba, I.I.; Moran, C.A. Expression of PD-1 and PD-L1 in thymic epithelial neoplasms. Mod. Pathol. 2017, 30, 826-833. [CrossRef] [PubMed]

24. Duan, J.; Liu, X.; Chen, H.; Sun, Y.; Liu, Y.; Bai, H.; Wang, J. Impact of PD-L1, transforming growth factor- $\beta$ expression and tumor-infiltrating $\mathrm{CD}^{+} \mathrm{T}$ cells on clinical outcome of patients with advanced thymic epithelial tumors. Thorac. Cancer 2018, 9, 1341-1353. [CrossRef]

25. Yokoyama, S.; Miyoshi, H.; Nakashima, K.; Shimono, J.; Hashiguchi, T.; Mitsuoka, M.; Takamori, S.; Akagi, Y.; Ohshima, K. Prognostic value of programmed death ligand 1 and programmed death 1 expression in thymic carcinoma. Clin. Cancer Res. 2016, 18, 4727-4734. [CrossRef] [PubMed]

26. Funaki, S.; Shintani, Y.; Fukui, E.; Yamamoto, Y.; Kanazaki, R.; Ose, N.; Kanou, T.; Minami, M.; Mori, E.; Okumura, M. The prognostic impact of programmed cell death 1 and its ligand and the correlation with epithelial-mesenchymal transition in thymic carcinoma. Cancer Med. 2019, 8, 216-226. [CrossRef] [PubMed]

27. Arbour, K.C.; Naidoo, J.; Steele, K.E.; Ni, A.; Moreira, A.L.; Rekhtman, N.; Robbins, P.B.; Karakunnel, J.; Rimner, A.; Huang, J.; et al. Expression of PD-L1 and other immunotherapeutic targets in thymic epithelial tumors. PLoS ONE 2017, 12, e0182665. [CrossRef]

28. Padda, S.K.; Riess, J.W.; Schwartz, E.J.; Tian, L.; Kohrt, H.E.; Neal, J.W.; West, R.B.; Wakelee, H.A. Diffuse high intensity PD-L1 staining in thymic epithelial tumors. J. Thorac. Oncol. 2015, 10, 500-508. [CrossRef] [PubMed]

29. Kondo, Y.; Monden, Y. Therapy for thymic epithelial tumors: A clinical study of 1320 patients from. Jpn. Ann. Thorac. Surg. 2003, 76, 878-884. [CrossRef]

30. Yokohama, S.; Miyoshi, H. Thymic tumors and immune checkpoint inhibitors. J. Thorac. Dis. 2018, 10, S1509-S1515. [CrossRef] 